Neural, Parallel, and Scientific Computations 28(2020), No.3, 182 - 197

\title{
TYPE-2 FUZZY BASED TESSERACT TEXT RELATIVE RECOGNITION ALGORITHM FOR NUMBER PLATE IDENTIFICATION
}

\author{
G.KAVITHA ${ }^{1}$ AND ANGELIN PEACE PREETHI.A ${ }^{2}$ \\ ${ }^{1}$ Department of Electronics and Communication Engineering \\ Government College of Technology, Salem, India \\ Email : kavitha@gcesalem.edu.in \\ ${ }^{2}$ Department of Electronics and Communication Engineering, \\ Karpagam College of Engineering, Coimbatore \\ Email : 3jsngl@gmail.com
}

Corresponding author : Angelin Peace Preethi.A

\begin{abstract}
Automatic number plate authentication is a mass tracking system that captures vehicles' images and authenticates their license number. Number plate detection can be used in many applications such as electronic payment methods (toll payment, parking payment), freeway, arterial monitoring systems for traffic monitoring, and stolen vehicles. Several methods have been proposed in previous studies to solve the following challenges (location size, size, color, font, opacity, gradient, etc.) by retrieving information from the captured image. However, each method has its drawback between the accuracy of the detection and the processing speed. This ambiguous inference system only gives an estimate of the limit plate of the clear hypothetical system answer. These methods work at different times for a different number of models. The Type2 Fuzzy based TesseractText Relative Recognition Algorithm (TTRR) obtains the image dataset from the JSON (JavaScript Object Notation) file and processes it through the link in the dataset. In the proposed detection, the method uses ambiguous logic classifiers that help identify plates and characters with excellent accuracy and processing speed. Compared to many significant contributions, Type2 Fuzzy based TTRR yields better results.
\end{abstract}

Key Words and Phrases: Fuzzy Inference System, Number Plate Recognition, Number Plate Detection, Character Segmentation, and Character Recognition.

Received July 21, 2020

www.dynamicpublishers.org;

https://doi.org/10.46719/npsc20202833.
1061-5369 \$15.00 C Dynamic Publishers, Inc. www.dynamicpublishers.com; 


\section{INTRODUCTION}

Pattern recognition and image processing are the vast areas of research in the current scenario. Many researchers around the world have attempted to automate processes in the past three decades in these areas. In recent years, the number of vehicles has increased significantly. An increased number of vehicles urges the need to focus on effective road traffic management. Control of the vehicle should be done because of several reasons, mainly security issues. This requires a management vehicle for intelligence work.

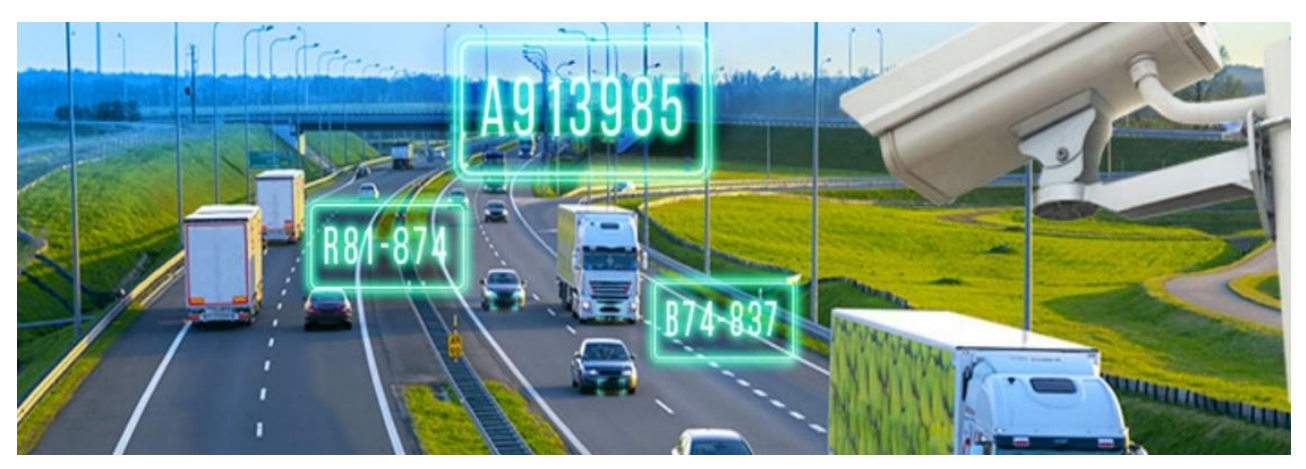

Figure 1 Number Plate Detection [21]

Each vehicle carries a number plate as a unique identifier of the vehicle. Manual recording of vehicles is time-consuming, costly, and not efficient. Therefore, it would always be beneficial to automate the process of vehicle number plate authentication. The integration of various aspects of information technology in the modern world has led to the concept of processing automotive information system resources. Due to an autonomous information system, the data makes no sense, and the vehicle information must be reformed between the information and the information system. It can be done by humans working in respective companies or by specialized smart devices, which allow vehicles to recognize number plates in real-world situations. Smart devices are specified that detect the number of plates in the vehicle system.

A digital image is obtained by converting it from a continuous analog image and stored in digital form. The process of converting an analog image to a digital one is called sampling and quantization. The image's continuous function is represented by a limited set of discrete values or observations using a digitization process. For continuous images, the processing of the digitized coordinate value of $f(X, Y)$ is called sampling, and the digitized amplitude or intensity value is 
called quantization. For example, a digital image representing the discrete value of each coordinate point of the pixel's intensity value is defined. If the analog signal is expressed as $\mathrm{X}$ (D), it will be continuous in time and range. Here, the sampling process's result is still a continuous range of time but a distinct signal. A digital signal is encoded by sample values from a sample data or signal and generates defined values.

This work is analyzed as follows. In section 2, the related works in the field are introduced and disussed to justify the contribution of this work. section 3 presents the materials and methods considered to implement the proposal. Next, section 4 shows and analyzes the main achieved results. Finally, in section 5, the main conclusions of the work are sum up.

\section{RELATED WORK}

The number of vehicles used in life is growing multifold. Many accidents have led to a linear increase in crime rates, increasing the number of stolen vehicles, violating traffic rules, and entering restricted areas. In such a busy world, theatrical recognition plays a vital role in identifying any vehicle license plate [1]. As the fastest growing mode of transport (vehicle) globally, it is difficult to track all of these vehicles and detect crimes. Therefore, it is essential for the authorities associated with [2] to maintain these vehicles' routes.

Detects and approves numberplates from a single frame on many vehicles should be made possible. The system consists of two steps: plate number detection and identification. It will work with the license plate from Spain for the exams [3]. Traffic control is a serious and significant issue for most vehicles, depending on the day and all day in the world. Sometimes it is difficult to determine the number plate violates traffic rules. The excellent model for vehicle positioning and the positioning plate is shown in color [4].

Since the structure and writing style are not the same, the group's design varies from one country to another. The license Plate Authentication System for Vehicle Authentication is a prevalent and challenging task. Unlike India, the vehicle owner's identity and the country that controls the most populous road traffic is a significant issue [5]. Powerful technology automated license plate authentication system for automated license plate detection and vehicle identification is to be implemented. [6]. 
The vehicle panel system is designed to handle different weather and lighting systems, challenging different traffic conditions and high-speed vehicles. It is a real-time, accurate hardware operating system [7] and a reliable and innovative way to solve various problems. Testing and certification of computer and visual information will be shared. The traditional detection method used in real-world [8] locations is used for horizontal boundary advice that does not change the text.

It is essential to find the images captured to the propulsion vehicles in real time so that they can be used for various purposes, such as detecting and understanding the movement of vehicles and other vehicles on the road in a specific place through analysis of the density of the number of this type of vehicles. Any traffic signal in a particular area, based on previous knowledge, is a reasonable assumption that the vehicle will go out [9]. The automatic license plate authentication system for registration number detection is an intelligent system that verifies image/video data and validates valid license plates [10].

According to research, there is no single way to find license plate authentication and license plate authentication from different angles. Standard layout [11] license plates are viewed from many angles. In addition to these displacements, the camera and the vehicle differ and play a vital role in the distance between the position of the plate [12] and the captured image.

Automatic license plate detection can help solve the problem of difficult parking and flow control. The system is designed using image processing and machine learning. The new system improves innovation in low light conditions and high output levels. It uses automotive image [13] quality, such as dark gray and standard to get it started working. The importance of being a smart transportation system has attracted a lot of attention in recent years of development. The automatic, fast, accurate, and powerful license plate certification system has become a requirement for traffic control and traffic law enforcement [14].

In doing so, one can read whether the vehicle passes the authentication verification by

comparing it with a particular campus vehicle's license plate and the pre-defined list of approved vehicles. To effectively extract these license plate numbers, we are trained to use modifications to our model and obtain a better output [15]. Traditionally, the system has handled this problem 
with continuous single-level detection in the image pyramid. Although this method simplifies the training process, as much evaluation as possible is required to consider the scale, leading to a linear increase in running and scale reviews [16].

Most of the existing systems achieve better performance, mainly considering the re-identified public vehicle appearance, rather than ignoring different vehicle identifiers such as license plates [17]. Therefore, a significant expansion is introduced by deploying its front and subgroups, reducing search space, and improving its output by rule-based local search [18] and genetically modifying it into a semi-hybrid category.

An SVM (Supported Vector Machine) is used to determine whether the license plate is in the candidate area based on negative and positive examples. The final license plate location is approved [19]. Finally, the Optical Character Recognition (OCR) segmentation [20] characters and number plate currently used to identify numbers in the NP area will be transmitted from the candidate area to the system[22-24].

\section{MATERIALS AND METHODS}

The Type-2 Fuzzy-Based Tesseract Text Relative Recognition of this system is specially designed to recognize vehicles' license plates. First of all, the system needs to be trained on some collected number plate data and cross-validation and repeat that process until the machine gets learned. When the machine-learned successfully, then further processing will take place. The vehicle's image has acquired a different color sets and different font styles. They are then fed to the first computer code reproduced grayscale image — and then extracted with the hidden region of the highest likelihood of the digital board that it can be used for further processing. There have been many robust approaches for recognizing letters and numbers on the license plates after passing the number plate detection process. Areas have now been interested in the characters and digital scanning coordinates, further changing the bounding box. The output is stored in a computerized program, and for each iteration, the result is entitled to include all the bits of the selected number of plates. Whenever the results meet a predetermined condition, the amount of computer code terminates the program display, so that the next image will be executed for processing. 


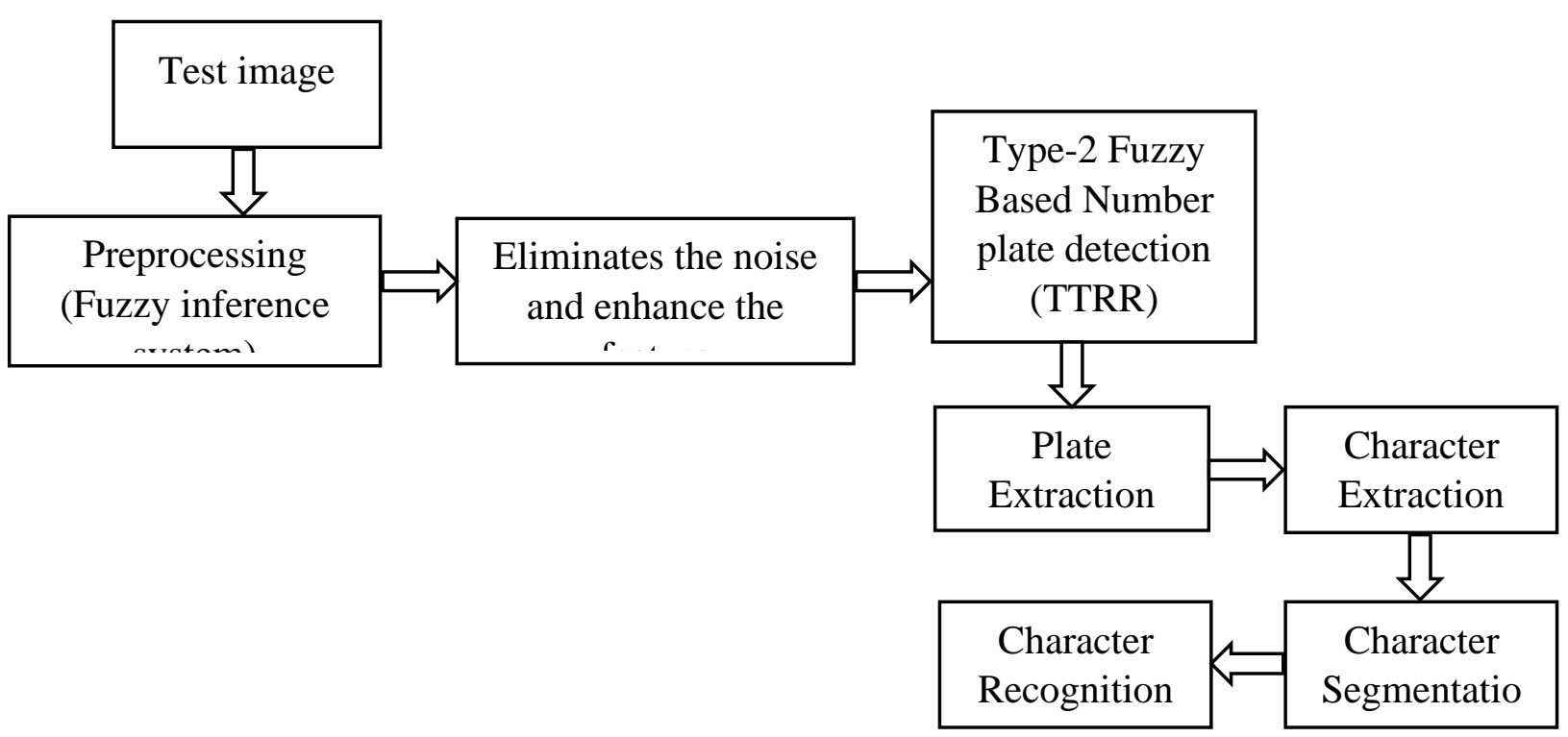

Figure 2 Proposed System Block Diagram

Figure 2 shows the input(Test) image; the size used to remove some noise from the preprocessing is also redesigned to make the manipulation image appropriate. After preprocessing, the plate extraction takes place, followed by character segmentation and detection. Then detect character and number recognition.

\subsection{Preprocessing}

The stage of image pre-processing constitutes a crucial stage since it aims to adequately prepare the image so that the remaining stages can be successfully carried out. Pre-processing involves grayscale conversion and binarization using material development technology. The Fuzzy Inference System improves the initial processing input image quality to prepare it for the system's next phase. Take images from the web and then remove noise and contaminants from the image. The edges and noise both represent a variation in intensity; usually, the edge has a considerable variation between adjacent pixels, compared to additive noise.

$$
\nabla_{N}(x, y)=(x, y-1)-I(x, y)
$$

The two necessary components of fuzzy systems are fuzzy sets and operations on fuzzy sets. Fuzzy logic defines rules, based on combinations of fuzzy sets by these operations. $X=\{x\}$ Defined by enumerating all elements $x \in X$ 


$$
\mathrm{A}=\left\{x_{1}, x_{2}, x_{3}, \ldots . x_{n}\right\}
$$

Filtering and Smoothing in Fuzzy Inference System:

A function can express membership and to compute the correlation term additional rule:

$$
\mathrm{A}=\left\{\left(\mathrm{x}, \mu_{A}(x)\right) \mid x \in X\right\}
$$

Where $\mu_{A}(X): X \rightarrow[0,1]$.

In Image Processing, usually greyscale images are used due to their greater simplicity compared to color images. Apart from this, in general, images usually present some undesired noise that needs to be removed. Besides, for this particular application, where number plates are the target, it is interesting to highlight the image. These certain features characterize number plates so that they can later be more easily detected.

\subsection{Number Plate Extraction Using CharacterFeatures}

This system is used for the first time and probably at the most critical stage. It is at this point that the position of the digit plate is determined. The input is the vehicle's image, and the output is the number plate. License plate extraction methods based on locating its characters have also been proposed. These methods examine the image of the characters. If the characters are found, their region is extracted as the license plate region. Instead of directly using the properties of the license plate, the algorithm tries to find all character-like regions in the image. It is achieved by using a region-based approach. Regions are enumerated and classified using a neural network. If a linear combination of character-like regions is found, the license plate's presence is assumed.

\section{Number Plate Extraction Based Character Features Define a Fuzzy Set:}

Step1: It may only be a limited set.

Example - Set of one digit even numbers, i.e., $A=\{2,4,6,8\}$.

A Fuzzy set is defined as a property satisfied by its members. 
$A=\{x \mid P(x)\}$, where p denoted as the property of $x$.

Example $-A=\{x \mid 0<x<9\}$ for $x \in X$, where $x$ is the data.

A Fuzzy set is also defined by the characteristics of the Extraction function (EA)

Step2: It declares which elements of the universal set $(\mathrm{X})$ are members of the set and which are not

$\mathrm{EA}(\mathrm{x})=1$ for $\mathrm{x} \in \mathrm{A}$

0 for $x \notin A$

It needs to determine all related areas in the image, using the concept of the Type2 Fuzzy based TTRR method. Other methods, like edge detection and morphological processing, can also be explored. Type2 Fuzzy based TTRR can help us connect the foreground area of the group and the label. The pixels are connected to another if they all have the same value and are adjacent to each other. If two straight lines are parallel within a certain range, and the number of the connected objects between them is similar to the characters, the area between them is considered the license plate area.

\subsubsection{Character Segmentation Using Extracted Features}

Since all character pixels do not have the same importance in distinguishing the character, a feature extraction technique that extracts some features from the character is an excellent alternative to the grey-level template matching technique. It reduces the processing time for template matching because not all pixels are involved. It also overcomes template matching problems if the features are strong enough to distinguish characters under any distortion. The extracted features form a feature vector compared with the pre-stored feature vectors to measure the similarity. The character is scanned along the central axis. The center axis is the moment of the connection between the upper limit horizontal center moment and the lower limit horizontal center-feature vectors generated by binary characters are extending horizontally and vertically.

\section{Type-2 Fuzzy Set Based Character Segmentation}


This section defines type-2 fuzzy sets and some critical associated concepts. By doing this, a simple collection of mathematically well-defined terms is provided that will let us effectively communicate about type-2 fuzzy sets.

\section{Doing this for all $x \in X$}

According to classical set theory, a subset A of a set $\mathrm{X}$ can be defined by its characteristic function $x_{A}$ as mapping the elements of $X$ to the set as $\{0,9\}$ as

$$
x_{A}: X \rightarrow\{0,9\}
$$

The statement is true $x_{A}=1$,

$$
x_{A}(t)=\{i f(a \leq t \leq b
$$

The membership function of a fuzzy set is a generalization of the indicator function in classical sets. It is a graphical representation of the magnitude of participation of each input. It is defined as a membership degree function

$$
\begin{gathered}
\mathrm{A}=\left\{\left(x, \mu_{A}(X)\right) \| x \in X\right\} \\
\mu_{A}(x)=0, \text { for } \mathrm{x}<a
\end{gathered}
$$

Characters are recognized using a related method i.e. New technology Type2 Fuzzy based TTRR design. All utensils by this Type2 Fuzzy based TTRR are located on the number plate of the region's vehicle input image extracted and classified.

\subsubsection{Type-2 Fuzzy Based Character recognition}

A Type2 Fuzzy based TTRR system can be developed with a highly customizable sample authentication machine that can detect patterns from possible formats. The structure was said to be carefully selected for use only, but to ensure that accreditation could be achieved at an acceptable time. This phase brings the actual recognition in to play. In this phase, extracted character regions are recognized. After each character is segmented from the number plate, the final operation is character recognition. 


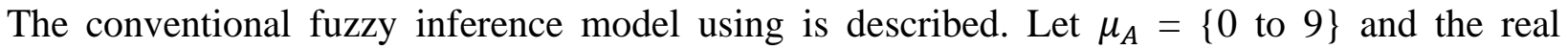
numbers let $\mathrm{x}=\left\{x_{1}, x_{2}, \ldots x_{n}\right\}$ and $\mathrm{y}$ represent as display the recognized output.

Where

$\mu_{A} \in \mathrm{X}$ for $\in x_{n}$ and

Then, the output y of the type-2 fuzzy method is obtained as

$$
\mathrm{y}=\frac{\sum_{0}^{n} \mu_{i} w_{i}}{\sum_{1}^{n} \mu_{t}}
$$

If the fuzzy function is used, then is expressed as $\mu_{A} \in \mathrm{X}$

$\mu_{i}(\mathrm{X})=\exp \left(-\frac{1}{2}\left(\frac{x_{i}-t_{i}}{w_{i}}\right)\right.$

Where

$w_{i}$-words, the processing image.

$\mu_{t}-$ denoted as time.

The input to this stage is the characters extracted from the number plate. The output of this stage is the recognized alphanumeric characters displayed on the number plate. Character recognition may have some challenges due to the camera's zoom ratio, non-uniform font size, and font type. Extracted alphanumeric characters may be broken and may contain some noise. Researches have used various techniques like artificial neural networks, support vector machine, template matching, etc. in literature. Each extracted character is separately recognized in the proposed system. Then, Type2 Fuzzy based TTRR technology is applied to the corresponding writing area to complete the task of proposing Type2 Fuzzy based TTRR effectively.

\section{RESULT AND DISCUSSION}

The main objective of this chapter is the performance analysis and verification of the proposed system. The multi-fold cross-validation is employed for testing the proposed algorithm. In cross-examination, the data set is divided into two subgroups. A particular subset of the 
database is used for training the system and is called trained data. Another subset is used to test the system and is called test data. The experimentations are performed on different partitions of the dataset. As the related technique is used, the result should point to two training and test data sets.

\begin{tabular}{|c|c|}
\hline Parameter & Value \\
\hline Data Set & JSON \\
\hline Trained Data & 300 \\
\hline Test Data & 200 \\
\hline Total Logs & 3 million \\
\hline Platform & Python \\
\hline Tool & jupyter notebook \\
\hline
\end{tabular}

Table 1 shows the resource needed in the Type2 Fuzzy based TTRR system,and it has been implemented to simulate jupyter notebooks connected to standard data sets.

Figure 3 shows the comparison of proposed and existing methods. The existing methods Support Vector Machine (SVM) in 67\%, Vehicle License Plate Detection Algorithm (VLPDA) in 70\%, Vessel Plate Number Recognition System (VPNRs) in 74\%, Deep Learning-Based Algorithm (DLA) in 83\%, and the proposed Tesseract text relative recognition (TTRR) in $89 \%$.

The proposed Type2 Fuzzy based TTRR using figure 5shows that the value of precision is $84 \%$, recall is $72 \%$, and Error Rate is $34 \%$, and speed $89 \%$.

It is observed that the incorrectly recognized images are there due to misclassification. Misclassification can occur when identification is different from that of extracted characters. The failure recognition analysis in figure 6 shows that the existing methods SVM 5 in sec character recognition, VLPDA 8 in sec character recognition, VPNRs 4 in sec character recognition, DLA 
7 in sec character recognition, Type 2 Fuzzy based TTRR takes time with 1 in sec character recognition.

\section{Character Recognition Accuracy}

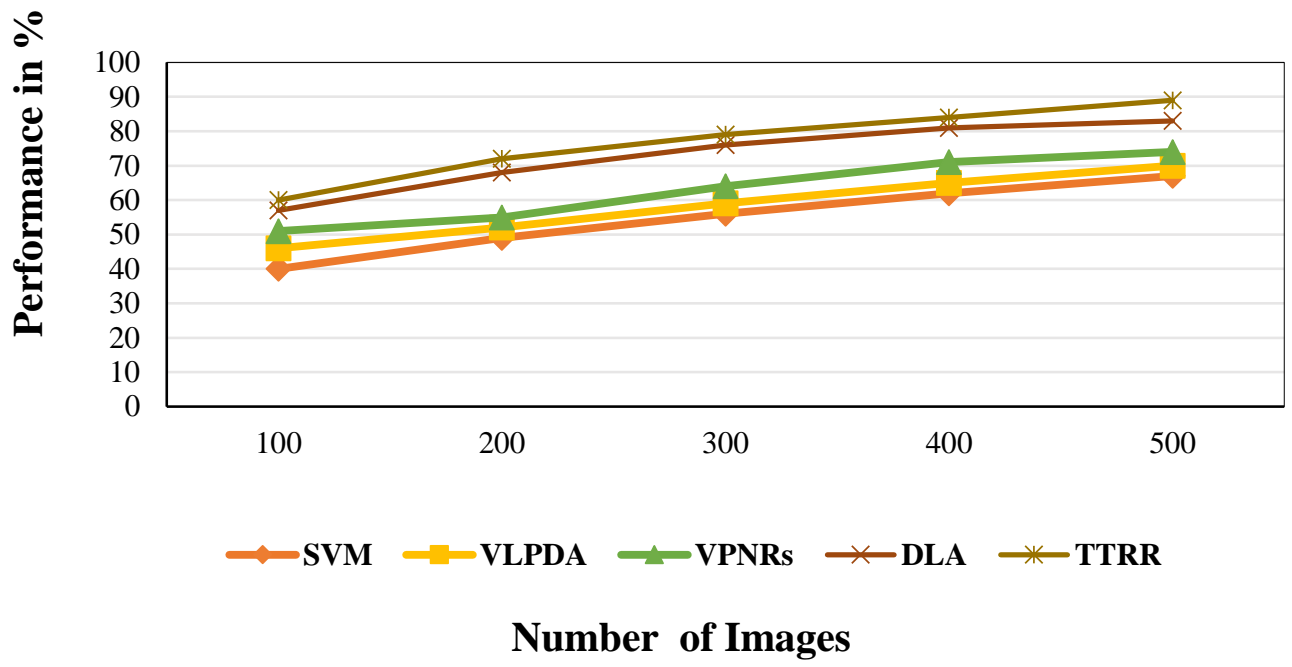

Figure 3 Analysis of Character Recognition Accuracy

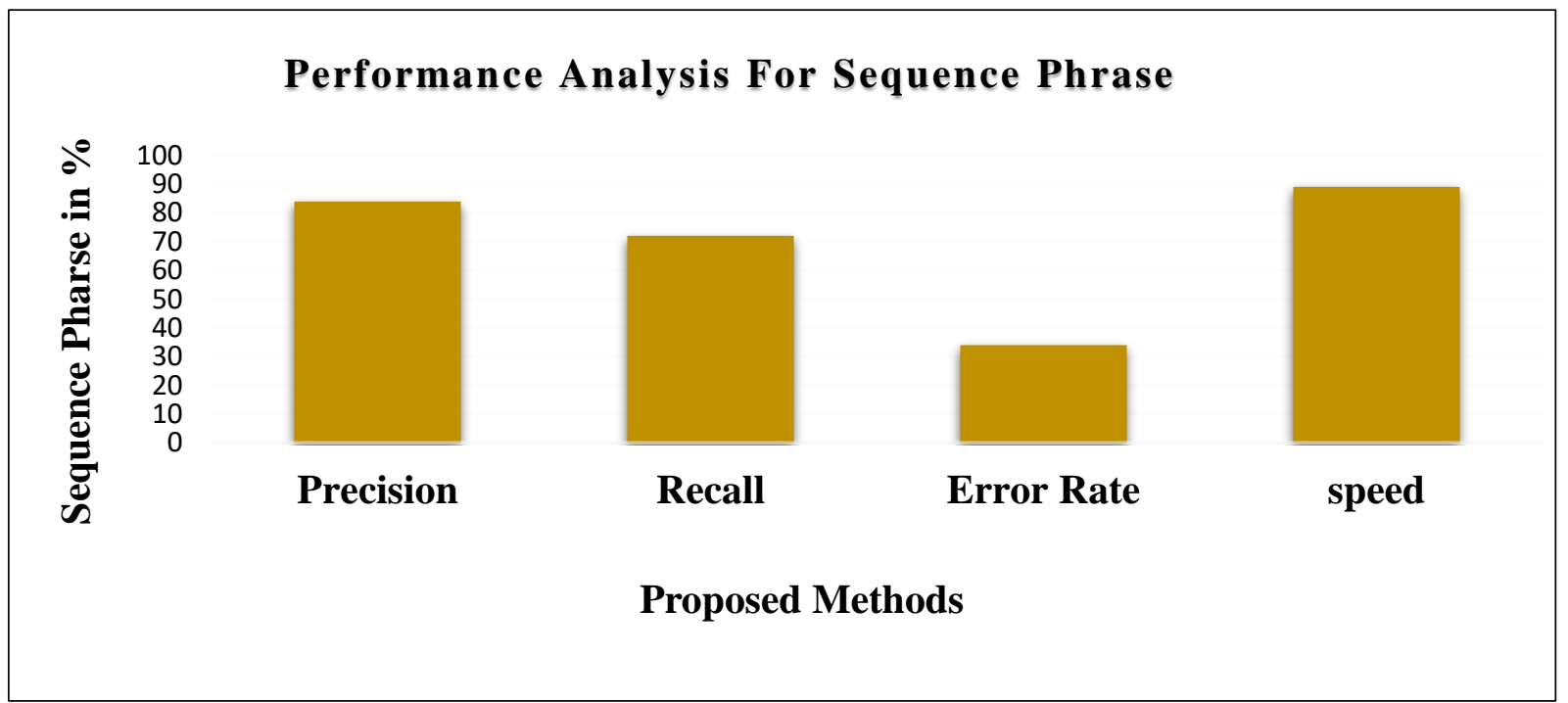

Figure 4 Performance Analysis in Sequence Phrase 


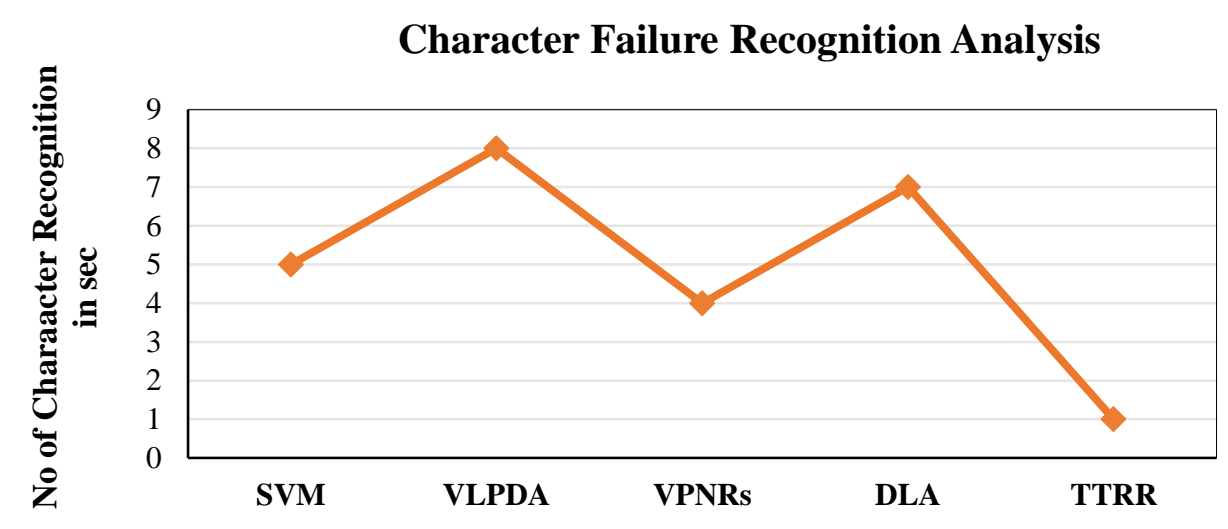

Figure 5 Character Failure Recognition Analysis

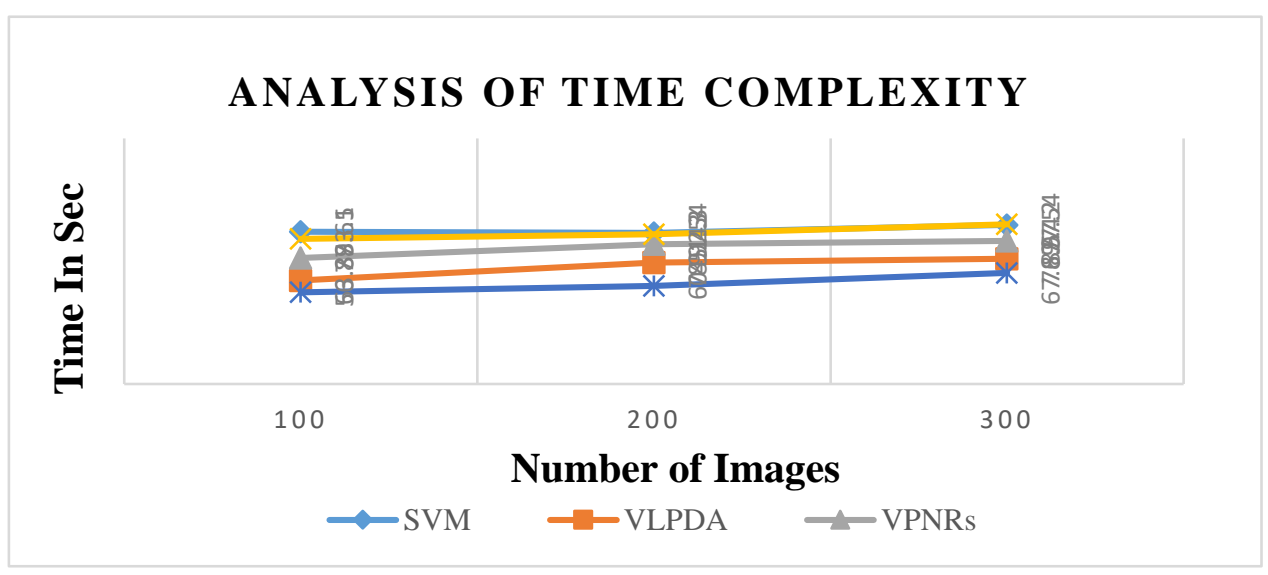

Figure 6 Analysis of Time Complexity

Figure 6 describes time complexity analysis shows that the SVM is 97.2 in ms, VLPDA in $76.5 \mathrm{~ms}$, VPNRs in $87.4 \mathrm{~ms}$, DLA in $97.54 \mathrm{~ms}$, and proposed Type2 Fuzzy based TTRR is 67.87 with ms.

\section{CONCLUSION}

Automatic vehicle number plate detection and authentication are vital technologies for most traffic-related applications. Many methods and technologies are being developed for digitization and approval. Few research results have solved problems in number plate recognition. Type- 2 Fuzzy Based Number plate accreditation involves three main stages: plate detection, writing division, and character recognition. Segmentation and number plate detection applied to the plate 
number are fundamental processes. The area where the number plate is extracted from the vehicle to detect any number of plates used. Number plate recognition is done efficiently so that each character is extracted and then all of the characters on the number plate are segmented. Type- 2 Fuzzy Based TTRR character segmentation and recognition accuracy of the system is based on performance. Type 2 Fuzzy based TTRR performance in the analysis of accuracy is $89 \%$, analysis of time complexity is 67.87 with $\mathrm{ms}$, precision value is $84 \%$, recall is $72 \%$, and the error rate is $34 \%$. Speed is $89 \%$ in failure recognition analysis, Type2 Fuzzy based TTRR takes 1 in sec of the time.

\section{REFERENCES}

[1]. Babu, K. M., \& Raghunadh, M. V. (2016). Vehicle number plate detection and recognition using the bounding box method. 2016 International Conference on Advanced Communication Control and Computing Technologies (ICACCCT), doi:10.1109/icaccet.2016.7831610.

[2]. Boliwala, R., \&Pawar, M. (2016). Automatic number plate detection for varying illumination conditions. 2016 International Conference on Communication and Signal Processing (ICCSP). doi:10.1109/iccsp.2016.7754224.

[3]. Menon, A., \&Omman, B. (2018). Detection and Recognition of Multiple NumberPlateFrom Still Images. 2018 International Conference on Circuits and Systems in Digital Enterprise Technology (ICCSDET). doi:10.1109/iccsdet.2018.8821138.

[4]. Naaman Omar Yaseen,Salim Ganim Saeed Al-Ali,AbdulkadirSengur (2019) An Efficient Model for Automatic Number Plate Detection using HOG Feature from New North Iraq Vehicle Images Dataset, 2019 IEEE, pp - (1-6).

[5]. Choudhury, A., \& Negi, A. (2016). A new zone based algorithm for detection of numberplate from Indian vehicle. 2016 Fourth International Conference on Parallel, Distributed, and Grid Computing (PDGC). doi:10.1109/pdgc.2016.7913178.

[6]. Yaseen, N. O., Ganim Saeed Al-Ali, S., \&Sengur, A. (2019). Development of New Anpr Dataset for Automatic Number Plate Detection and Recognition in North of Iraq. 2019 1st International Informatics and Software Engineering Conference (UBMYK). doi:10.1109/ubmyk48245.2019.8965512. 
[7]. Panahi, R., \&Gholampour, I. (2017). Accurate Detection and Recognition of Dirty Vehicle Plate Numbers for High-Speed Applications. IEEE Transactions on Intelligent Transportation Systems, 18(4), 767-779. doi:10.1109/tits.2016.2586520.

[8]. Huang, S., Xu, H., Xia, X., \& Zhang, Y. (2018). End-to-End Vessel Plate Number Detection and Recognition Using Deep Convolutional Neural Networks and LSTMs. 2018 11th International Symposium on Computational Intelligence and Design (ISCID). doi:10.1109/iscid.2018.00051.

[9]. Santra, S., Roy, S., Sardar, P., \&Deyasi, A. (2019). Real-Time Vehicle Detection from Captured Images. 2019 International Conference on Opto-Electronics and Applied Optics (Optronix). doi:10.1109/optronix.2019.8862323.

[10]. Aung, K. P. P., Nwe, K. H., \& Yoshitaka, A. (2019). Automatic NumberPlate Detection System for Myanmar Vehicle NumberPlates. 2019 International Conference on Advanced Information Technologies (ICAIT). doi:10.1109/aitc.2019.8921286.

[11]. Khin, O., Phothisonothai, M., \&Choomchuay, S. (2017). NumberPlate Detection of Myanmar Vehicle Images from Dissimilar Angle Conditions. 2017 21st International Computer Science and Engineering Conference (ICSEC). doi:10.1109/icsec.2017.8443908.

[12]. Khin, O., Phothisonothai, M., \&Choomchuay, S. (2017). NumberPlate Detection of Myanmar Vehicle Images Captured from the Dissimilar Environmental Conditions. 2017 International Conference on Advanced Computing and Applications (ACOMP). doi:10.1109/acomp.2017.31.

[13]. Babbar, S., Kesarwani, S., Dewan, N., Shangle, K., \& Patel, S. (2018). A New Approach for Vehicle Number Plate Detection. 2018 Eleventh International Conference on Contemporary Computing (IC3). doi:10.1109/ic3.2018.8530600.

[14]. Dias, C., Jagetiya, A., \&Chaurasia, S. (2019). Anonymous Vehicle Detection for Secure Campuses: A Framework for NumberPlate Recognition using Deep Learning. 2019 2nd International Conference on Intelligent Communication and Computational Techniques (ICCT). doi:10.1109/icct46177.2019.8969068.

[15]. Kakani, B. V., Gandhi, D., \& Jani, S. (2017). Improved OCR based automatic vehicle number plate recognition using features trained neural network. 2017 8th International Conference on Computing, Communication, and Networking Technologies (ICCCNT). doi:10.1109/iccent.2017.8203916. 
[16]. Efficient Scale-Adaptive NumberPlate Detection System. (2018). IEEE Transactions on Intelligent Transportation Systems, 1-13. doi:10.1109/tits.2018.2859035.

[17]. Liu, X., Liu, W., Mei, T., \& Ma, H. (2018). PROVID: Progressive and Multimodal Vehicle Reidentification for Large-Scale Urban Surveillance. IEEE Transactions on Multimedia, 20(3), 645-658. doi:10.1109/tmm.2017.2751966.

[18]. Miyata, S., \& Oka, K. (2016). Automated numberplate detection using a support vector machine. 2016 14th International Conference on Control, Automation, Robotics, and Vision (ICARCV). doi:10.1109/icarcv.2016.7838653.

[19]. Yin, Zongyi et al. 'Intelligent Forecasts and Evaluation of Financial Risk Assets Based on Grey Markov Chain Model, Journal of Intelligent \& Fuzzy Systems, Volume 35, issue 3, 1 Jan. 2018, pp.2679 - 2684.

[20]. Ghosh, R., Thakre, S., \& Kumar, P. (2018). A vehicle number plate recognition system using region-of-interest based filtering method. 2018 Conference on Information and Communication Technology (CICT). doi:10.1109/infocomtech.2018.8722345.

[21]. Nguyen, Trong-Kha, Ly, Vu Duc, and Hwang, Seong Oun. 'An Efficient Neural Network Model for Time Series Forecasting of Malware', Journal of Intelligent \& Fuzzy Systems Volume 35, issue 6, 1 Jan. 2018, pp. $6089-6100$.

[22]. Thangallapally, S. K., Maripeddi, R., Banoth, V. K., Naveen, C., \&Satpute, V. R. (2018). E-Security System for Vehicle Number Tracking at Parking Lot (Application for VNIT Gate Security). 2018 IEEE International Students' Conference on Electrical, Electronics, and Computer Science (SCEECS). doi:10.1109/sceecs.2018.8546903.

[23]. Yap, Khong-Lim | Chong, Yung-Wey | Ko, Kwangman, Performance comparison between progressive mobility prediction using hidden Markov model and multipath transmission control protocol, Journal of Intelligent \& Fuzzy Systems, vol. 35, no. 6, pp. 5827-5836, 2018

[24]. https://globalsmtseasia.com/technology_news/artificial-intelligence-api-for-car-parkingmanagement-system-using-stm32f407-and-image-processing-usingopencv/\#: :text=Fig.\%204:\%20Block\%20Diagram\%20of\%20the\%20Open\%20CV\%20 Technique. 